\title{
La création du parc naturel marin de Martinique : entre concertation et fragmentation
}

\author{
Hubert Mazurek ${ }^{1, *}$, Louis Arreghini ${ }^{1}$, Caroline Cochet $^{2}$, Justin Daniel ${ }^{3}$, \\ Jean-Raphaël Gros-Désormeaux ${ }^{4}$ et Debra Pereira ${ }^{5}$ \\ ${ }^{1}$ Géographie, Aix-Marseille Université, IRD, UMR151 LPED, Marseille, France \\ 2 Droit, CNRS, Université des Antilles, UMR8053 LC2S, Fort-de-France, Martinique \\ 3 Sciences politiques, CNRS, Université des Antilles, UMR8053 LC2S, Fort-de-France, Martinique \\ ${ }^{4}$ Géographie, CNRS, Université des Antilles, UMR8053 LC2S, Fort-de-France, Martinique \\ 5 Sociologie de l'environnement, Aix-Marseille Université, IRD, UMR151 LPED, Marseille, France
}

Reçu le 16 septembre 2017. Accepté le 23 octobre 2018

Cet article relate la démarche de création d'un parc naturel marin aux Antilles. Il produit une analyse fine de l'implication des acteurs dans le processus de participation mis en place pour accompagner cette création. Il vient ainsi enrichir une série d'articles publiés dans la revue sur cette thématique de la participation, entendue comme modalité d'interaction et d'intégration à l'interface société-environnement. Il apporte un regard critique sur la démarche mise en œuvre, en explicitant des raisons de l'échec de l'implication de certains groupes. Cette analyse des interactions entre les pêcheurs et les autres parties prenantes concernées par la création de cette aire marine protégée propose en outre une autre vision des apports possibles de la participation.

La rédaction

Résumé - Le processus de concertation puis de création du parc naturel marin de Martinique a fait l'objet d'une analyse à partir de la participation de deux classes d'acteurs : les pêcheurs et les mouvements associatifs. Alors que l'objectif même du parc est de construire un consensus sur la nécessité de la conservation du milieu marin et d'y associer des outils de gestion concertée, la concertation a de fait fragmenté ces groupes d'acteurs. Le point de vue du législatif et du politique conforte cette analyse. Il montre les limites de ces approches et la nécessité d'une intégration plus efficace des secteurs sociaux et productifs pour la construction d'un projet territorial. La fragmentation des acteurs accroît de fait la vulnérabilité de la gouvernance de ces zones marines et littorales, à l'encontre des objectifs de protection et de conservation.

Mots-clés : environnement / gouvernance / aire marine protégée / association / pêche / Martinique

\begin{abstract}
The creation of the Marine Nature Reserve of Martinique: between preservation and fragmentation. The Marine Nature reserve of Martinique was established in 2017 by the French Ministry of the Environment following a dialogue process which lasted 5 years. This article makes a first analysis of this process on the basis of two groups of actors: the fishermen and the associative movements. While the objective of the Park is to build a consensus on the need to preserve the marine environment, associating tools for a joint management in the process, the dialogue in fact split up these groups of actors. Both types of actors were de facto excluded from the dialogue process; the fishermen because of poor communication which marginalized them and the integration of their knowledge in the discussion and the associations because of a substitution of their skills by those of the State services. The point of view of legislative and political actors consolidates this analysis; it shows the limits of these approaches towards a normalized participation process and the necessity of a more effective integration of the social and
\end{abstract}

\footnotetext{
*Auteur correspondant : Hubert.mazurek@ird.fr
} 
productive sectors for the construction of a territorial project. The fragmentation of the actors involved increases de facto the vulnerability of the governance of these marine and littoral zones, and runs counter to the protection and preservation objectives.

Keywords: environment / governance / protected marine area / association / fishing / Martinique

En septembre 2013, l'Agence des aires marines protégées (AAMP) et le ministère de l'Écologie, du Développement durable et de l'Énergie se fixent comme priorité d'étudier la faisabilité d'une création de parc naturel marin (PNM) dans les Antilles françaises ${ }^{1}$, sur le territoire marin de la Martinique ${ }^{2}$. Cette intention figurait déjà dans l' " arrêté du 13 avril 2012 relatif à la conduite de la procédure d'étude et de création d'un parc naturel marin en Martinique ${ }^{3}$ » signé par François Fillon, alors Premier ministre et ministre de l'Environnement ${ }^{4}$, peu avant l'élection présidentielle de 2012. Cet arrêté comporte trois articles: le premier donne autorité au préfet de Martinique pour la conduite de la procédure d'étude et de création du parc naturel marin de Martinique (PNMM); le second spécifie que les eaux sous juridiction française autour de la Martinique constituent le périmètre d'étude du projet; et le troisième liste les institutions en charge de l'exécution de cet arrêté. Les dispositions juridiques relatives à la création et à la gestion des parcs naturels marins sont énoncées dans le Code de l'environnement, dans sa partie législative ${ }^{5}$ et dans sa partie réglementaire ${ }^{6}$. Il est à noter que le code se focalise davantage sur les modalités de fonctionnement de l'aire marine que sur la procédure de création (concertation, information, déroulement). Il impose que le décret de création du parc naturel marin soit pris après réalisation d'une enquête publique ${ }^{7}$. Mais la consultation en Martinique comprendra également une phase de « concertation préalable à l'enquête publique ${ }^{8}$ ».

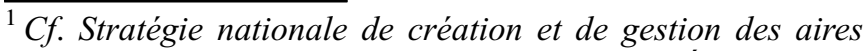
marines protégées. Synthèse du ministère de l'Écologie, du Développement durable et de l'Énergie, www.ecologiquesolidaire.gouv.fr/sites/default/files/Strat $\%$ C3\%A9gie\% 20nationale $\% 20 \mathrm{de} \% 20 \mathrm{cr} \% \mathrm{C} 3 \%$ A9ation $\% 20$ et $\% 20 \mathrm{de} \% 20$ ges tion $\% 20 \mathrm{des} \% 20$ aires $\% 20$ marines $\% 20$ prot $\% \mathrm{C} 3 \% \mathrm{~A} 9 \mathrm{~g} \% \mathrm{C} 3 \%$ A9es.\%20Synth\%C3\%A8se.pdf

${ }^{2}$ Cette étude s'inscrit dans le cadre du projet «Territoires et acteurs au prisme des aires marines protégées. Enjeux, synergies, contraintes (Terrimar)», financé par la Fondation de France, ${ }^{\circ} 00059169$.

${ }^{3}$ Journal officiel, 92, 18 avril 2012, 6980, texte $\mathrm{n}^{\circ} 3$.

${ }^{4}$ Ministère de l'Écologie, du Développement durable, des Transports et du Logement.

${ }^{5}$ Livre III, Titre III, Chapitre IV, section 2, article L. 334-3 et suivants.

${ }^{6}$ Article R. $334-27$ et suivants.

${ }^{7}$ Article L. 334-3.

${ }^{8}$ Conformément à l'ancien article L.121-16 en vigueur jusqu'au $1^{\text {er }}$ janvier 2017.
}

La mission d'étude a été mise en place en mai 2013. Dès la première réunion d'un comité de concertation convoqué par le préfet le 4 décembre 2013, les grandes lignes du parc ont été présentées selon un schéma désormais rodé au regard des parcs marins et des parcs naturels précédemment créés ${ }^{9}$. Ce comité et la mission ont insisté sur les enjeux de connaissances et de protection du milieu marin. Le débat a essentiellement porté sur le fonctionnement du parc (périmètre, conseil de gestion, plan de gestion). Enfin, l'enquête publique a été conduite en janvier et février 2017, pour donner lieu à un avis négatif, qui sera sans incidence sur la signature du décret de création le 24 mars $2017^{10}$ par la ministre de l'Écologie, du Développement durable et de l'Énergie. Le PNMM a donc été officiellement créé cinq ans après le premier arrêté et près d'un mois avant l'élection présidentielle française de 2017.

Ces cinq années se caractérisent par un curieux paradoxe: d'un côté, la mission déploie une intense activité de coconstruction, en accumulant des connaissances, en multipliant des réunions de concertation, en produisant des informations et en rédigeant des documents; de l'autre, les propositions d'orientation, le schéma d'organisation et de gestion initialement prévus ne varient guère. Entre le début et la fin de la concertation, beaucoup d'informations circulent, avec principalement pour effet d'éveiller la curiosité, tout en maintenant un flou sur les objectifs de ce parc et sur les compétences que le conseil détiendra sur un territoire marin qui, il faut bien le rappeler, est très dépendant des activités terrestres de l'île.

Cet article propose de jeter rétrospectivement un regard croisé sur cette période dédiée à l'information et à la concertation. L'objectif est de comprendre et de mettre au jour le jeu des acteurs, et d'anticiper les défis, contenus en filigrane dans les débats, que doit relever le conseil de gestion du parc. Il est clair, en effet, que le mode de communication dans le cadre des processus de concertation est déterminant pour le fonctionnement des

\footnotetext{
${ }^{9}$ Dix parcs marins ont été créés depuis 10 ans; voir le site www.aires-marines.fr.

${ }^{10}$ Décret n $^{\circ}$ 2017-784 portant création du parc naturel marin de Martinique, signé par la ministre le 24 mars 2017 en Martinique, daté du 5 mai 2017, Journal officiel, 108, 7 mai 2017 , texte $\mathrm{n}^{\mathrm{o}} 6$.
} 
instruments de gestion (Billé, 2006; Lequin, 2009). La façon dont sont générées et transférées les informations, utilisés les modes de consultation, menée la scénarisation des dialogues, et surtout convoqués les intervenants, influence probablement la compréhension du processus et par conséquent son appropriation. Par ailleurs, un processus de concertation doit permettre de combattre, au regard de l'objectif de conservation, la vulnérabilité des écosystèmes liée au jeu des acteurs et à la gouvernance des dispositifs. Nous nous intéresserons par conséquent aux interactions entre la mission d'étude de l'Agence des aires marines protégées - actuellement Agence française de la biodiversité (AFB) - et les usagers du milieu marin dans un cadre fortement marqué par des particularismes sociohistoriques et politiques. L'accent sera mis sur la concertation préalable à l'installation du PNMM en tant que catalyseur de fragmentations sociales. Ce regard nous semble utile pour améliorer les dispositifs de concertation des services de l'État, dans un contexte où la participation effective de la société civile devient une exigence pour la gouvernance locale.

\section{Méthodologie de travail}

Le programme Terrimar a démarré en janvier 2016. Son ambition était d'analyser, selon une démarche interdisciplinaire ${ }^{11}$, le processus de concertation mené dans la perspective de la création du PNMM. Ce travail a été mené en collaboration avec la mission du parc, qui a fourni l'ensemble de la documentation relative aux diagnostics préalables, aux listes de présences aux réunions ( 8 réunions thématiques publiques, 11 réunions de concertation publiques, 1 forum et 2 réunions de synthèses convoquées par le préfet) et les retranscriptions intégrales des débats de ces réunions (verbatim).

Les analyses menées dans le cadre de ce programme, dont une partie est utilisée pour cet article, ont porté sur cinq ensembles de données :

- Le contenu des verbatim de réunions et de la documentation produite par la mission; leur analyse fut réalisée principalement grâce au logiciel N'Vivo, elle a aussi permis de comparer les éléments de débat au fil du temps, la rhétorique argumentative des acteurs et les modifications des orientations;

- La documentation juridique;

- 15 entretiens, entre une et trois heures, auprès des institutions, des collectivités territoriales et de quelques associations très impliquées dans le processus ;

\footnotetext{
${ }^{11}$ Participent à ce projet : deux géographes et une sociologue de l'environnement du LPED, une juriste, un politiste, un géographe et une spécialiste des sciences de l'information et de la communication du LC2S.
}

- Des entretiens réguliers pendant plus d'un mois auprès des pêcheurs et de leurs associations locales (depuis Case-Pilote jusqu'au Prêcheur);

- Des entretiens semi-directifs, auprès d'une quinzaine $\mathrm{d}$ 'associations de protection de la nature et d'usagers de l'environnement (club de plongée, association sportive Kayak, de découverte, etc.), en utilisant la typologie diffusée par les services de la préfecture complétée par les associations inscrites au Journal officiel et non déclarées en préfecture. Ces entretiens ont été menés à l'aide d'un guide commun à toutes les associations; ils ont duré une heure minimum.

La démarche d'entretiens a été structurée de manière à mettre en lumière les perceptions et les représentations des acteurs vis-à-vis de la création du PNMM, en tentant de saisir leurs postures avant, pendant et après la procédure. Parallèlement, une analyse d'acteurs et de leurs objectifs a permis de situer les synergies et les antagonismes à partir des discours et des compétences (Godet, 1997; Gumuchian et al., 2003).

\section{Une mainmise de l'État sur le littoral martiniquais}

La définition du périmètre du parc a suscité de nombreux débats et plusieurs options ont été envisagées avant de s'en tenir à la projection initialement envisagée : l'ensemble des eaux sous juridiction française entourant l'île de la Martinique, soit une superficie de $47340 \mathrm{~km}^{2}$ (zone économique exclusive, ZEE) [Fig. 1]. La plupart des acteurs présents aux réunions étaient pourtant favorables à un parc plus limité, permettant une gestion plus efficace, surtout en période de disette budgétaire. Une bande littorale élargie aurait permis, selon plusieurs participants aux débats, une meilleure cohérence de la gestion avec les activités humaines. Mais, comme le souligne Féral (2011), cette délimitation fait partie de la tendance mondiale d'une mainmise de l'État sur les ZEE grâce à la création de zones de protection de très grande taille, au détriment de la normativité.

D'un point de vue juridique, la zone littorale de la Martinique est délimitée selon deux dispositifs réglementaires, reflétant les usages privilégiés des acteurs. Le premier correspond à la ligne des 50 pas géométriques $(81,2 \mathrm{~m})$, zone terrestre inaliénable et imprescriptible (sauf pour les zones urbaines), datant d'un arrêté de 1827, en situation de régularisation par l'agence du même nom ${ }^{12}$. La seconde correspond à une bande littorale relative au balisage et à la signalisation des 300 mètres $^{13}$; elle devient la limite du pouvoir communal de police spéciale en mer

\footnotetext{
12 www.agence50pas972.org.

${ }^{13}$ Arrêté du 27 mars 1991, Journal officiel, 101, 28 avril 1991, 5762, version consolidée au 29 octobre 2018.
} 


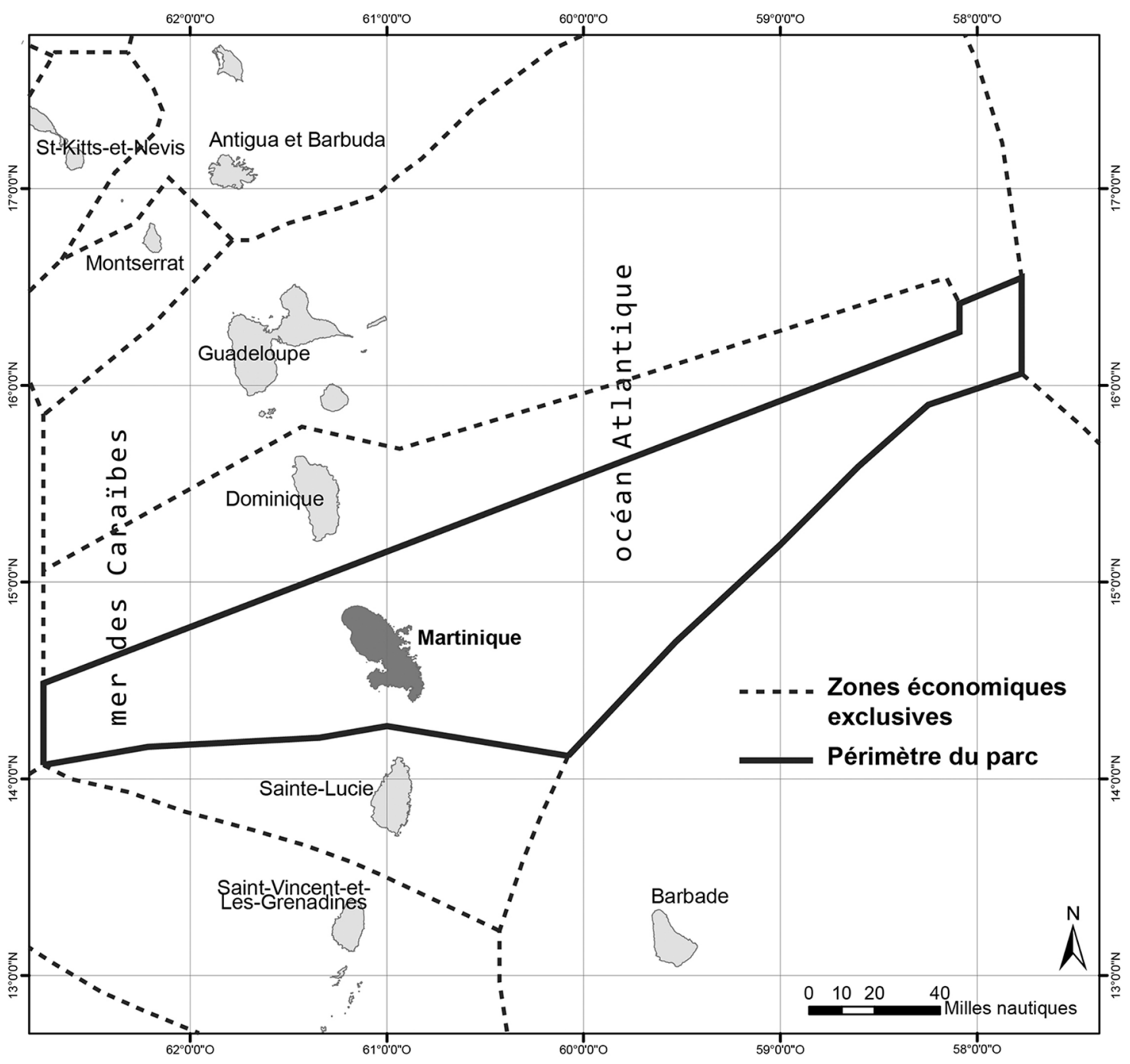

Fig. 1. Délimitation du parc naturel marin de Martinique, correspondant à la ZEE (source: d'après le ministère de l'Environnement [dossier de presse du 24 mars 2017]; réalisation: H. Mazurek).

pour les baignades et les activités nautiques pratiquées à partir du rivage avec des engins de plage et des engins non immatriculés, et aussi de l'usage des moteurs et la réglementation de la vitesse, ou la possibilité de pratiquer des sports nautiques comme le kitesurf ou le ski nautique. Cette limite est également imposée aux pêcheurs pour différencier la pêche côtière (senne, pêche artisanale ou traditionnelle) de la pêche motorisée (pêche hauturière). De plus, cette zone littorale est régie par des règlements très divers, découlant des situations locales et des particularismes du territoire: cantonnements de pêche, zones d'interdictions de pêche à cause d'une forte contamination au chlordécone, zones du port maritime de Fort-de-France, zones de réserve naturelle (Prêcheur et Caravelle), mangroves, etc. ${ }^{14}$ (Fig. 2).

\footnotetext{
${ }^{14}$ On peut avoir un recensement de l'ensemble de ces zones auprès de l'observatoire de l'eau de la Martinique: www. observatoire-eau-martinique.fr.
}

Les principaux enjeux liés à la conservation et la protection du milieu marin sont littoraux; la plupart des orientations mentionnées dans le décret de création le montrent aussi. Compte tenu de ce contexte, le programme Terrimar a concentré son analyse sur trois thématiques, dont quelques résultats significatifs sont exposés ci-dessus : le processus participatif du point de vue juridique et opérationnel, la participation du secteur de la pêche professionnelle, et celle des associations de protection de la nature et d'usagers de la mer.

\section{Droit à la participation et effectivité}

Si le décret de création du parc naturel marin (PNM) de Martinique est considéré comme une réussite pour l'équipe de l'Agence française pour la biodiversité en charge de la concertation et de la connaissance, les conclusions de l'enquête publique remettent en cause la procédure de concertation. La mission d'étude a 


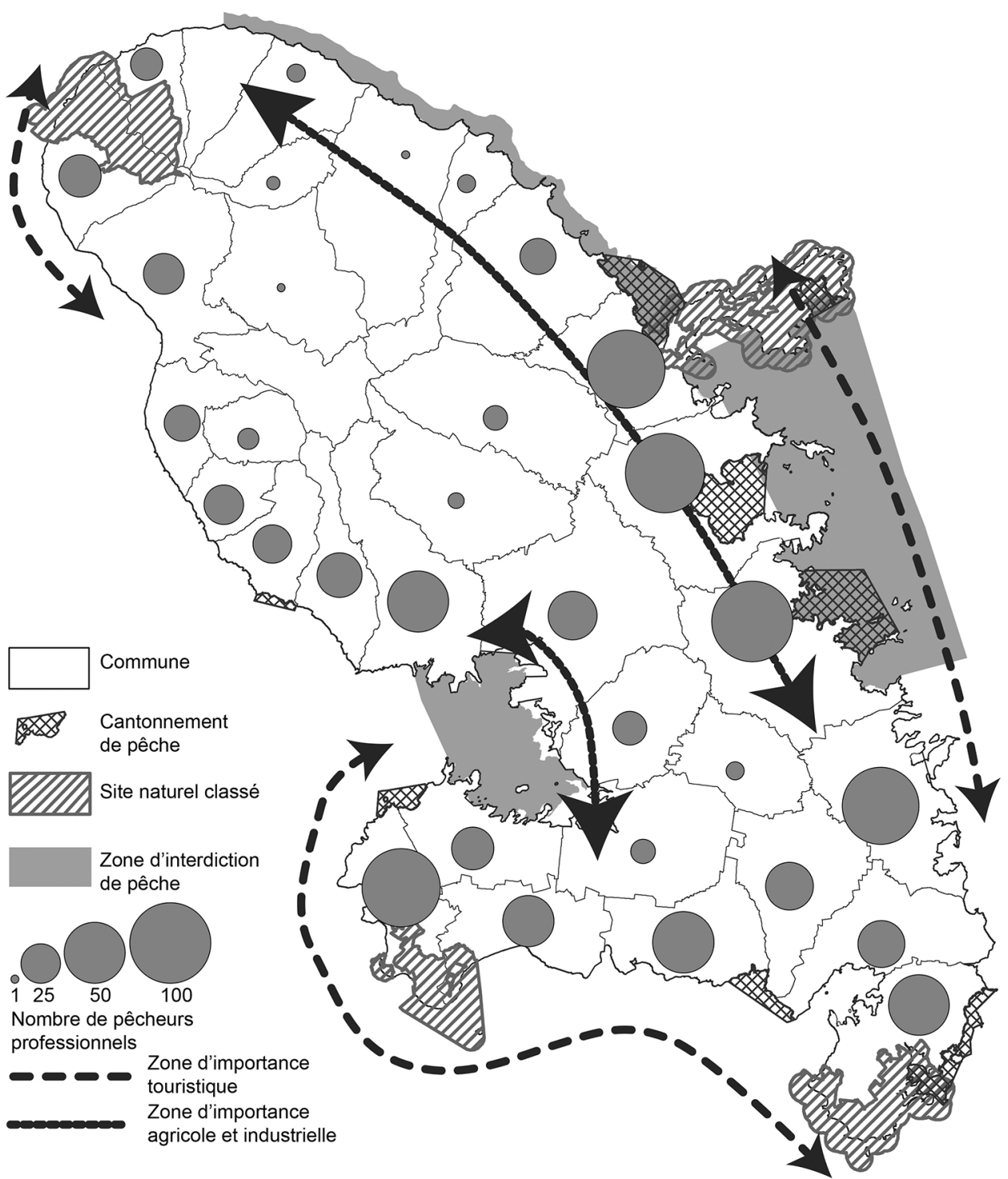

Fig. 2. Les enjeux liés à la conservation de la zone littorale: des interactions complexes entre activités, conservation et contamination (source: synthèse d'après les documents de diagnostics et les enjeux du PNMM, 2010-2016; réalisation: H. Mazurek).

notamment été critiquée du fait de la mobilisation insuffisante des élus de proximité en tant que relais dans la diffusion de l'information. Au regard des précédents constats, il apparaît que l'approche technico-administrative a donné priorité au respect de la procédure de cadrage du PNM (périmètre, orientations et gouvernance), probablement au détriment d'une véritable démarche de territorialisation (Micoud, 1991). Les deux phases de consultation du public mises en œuvre préalablement à la création du PNM de Martinique $^{15}$ n'ont connu qu'une faible mobilisation de l'ensemble des

\footnotetext{
${ }^{15}$ En application de l'article L.121-16 du Code de l'environnement dans sa version antérieure à janvier 2017 pour la concertation préalable, ainsi que des articles L.123-1 et suivants, et R.123-1 et suivants pour l'enquête publique.
}

acteurs concernés par le projet. L'effectivité de la participation n'est certes pas une exigence juridique mais bien un but visé ; l'effort d'information et d'organisation des réunions n'a pas été suffisant pour intéresser le public et l'inciter à s'impliquer. La communauté de pêcheurs, nous le verrons, n'a que très peu participé aux réunions de concertation et ne s'est que très faiblement manifestée lors de l'enquête publique. Le respect des règles procédurales applicables en la matière n'a donc pas suffi à susciter une participation effective. En l'absence de méthodologie prévue par les textes juridiques pour le déroulement de la concertation, l'équipe de l'Agence des aires marines protégées en charge de cette mission a suivi la stratégie et les orientations proposées en la matière par le ministère chargé de l'écologie. Mais malgré l'absence persistante des pêcheurs, aucun changement de stratégie 
ni aucune adaptation notable n'ont été tentés afin de déclencher l'implication de cette communauté d'acteurs. En ce qui concerne l'enquête publique, le rapport rendu par les commissaires-enquêteurs a souligné, entre autres, le défaut de prise en compte des conditions socioculturelles martiniquaises et du contexte local qui furent, à la période choisie pour son déroulement, défavorables à l'intervention du public dans la procédure. Il précise que la consultation s'est déroulée parallèlement à la mise en place d'une nouvelle collectivité territoriale en Martinique, c'est-à-dire une période sensible "ayant nécessité des élections et l'implication de tous et de toutes ». L'enquête publique s'est tenue à l'ouverture de la saison carnavalesque, temps de «relâchement» festif durant lequel «à quoi s'attendre en termes d'intérêt et de participation du public sinon à souhaiter une absence de participation"» (rapport d'enquête, p. 41-42.). Enfin le rapport relève que la procédure s'est superposée à une phase d'élections professionnelles des comités des pêches, ce qui a eu pour conséquence «une absence de communication officielle des marins pêcheurs et des aquaculteurs » (rapport d'enquête, p. 42). Ces conditions locales ont pu détourner l'attention du public du projet de PNM.

Il en résulte un fort questionnement quant à la qualité et à l'accessibilité de l'information diffusée concernant le projet ainsi qu'à la pertinence des conditions mêmes de mise en œuvre des procédures.

\section{Les enjeux de la pêche pour le parc naturel marin de Martinique}

Un des objectifs annoncés de l'AAMP concerne la gestion responsable des activités économiques et de loisirs. Plusieurs orientations ${ }^{16}$ répondent à cet objectif:

«En tenant compte du fort lien terre-mer, soutenir une gestion innovante et participative dans les projets de développement visant à concilier les différents usages et intégrant les services rendus par les écosystèmes marins [...] Engager les activités de tourisme, de sports et de loisir et les autres filières économiques dans des pratiques responsables et durables, participer à la formation des acteurs de la mer et inciter à la mise en place d'équipements adaptés notamment de mouillages et portuaires, tout en associant les collectivités territoriales [...] Soutenir la pêche côtière artisanale et l'aquaculture » (Décret de création du parc national marin de Martinique).

Le document de diagnostic réalisé lors de la phase d'étude (AAMP, 2010) précise dès le début du processus que la création d'une aire marine protégée dans un espace

\footnotetext{
${ }^{16}$ Les orientations ont fait l'objet de cinq débats spécifiques, avec des modifications importantes entre le résultat initial de la synthèse et la proposition présentée à l'enquête publique puis au décret.
}

où les activités humaines sont très présentes nécessite une structuration des filières dans le respect de la réglementation et une amélioration de toutes les pratiques maritimes dans un souci de protection et de préservation des milieux, en particulier du littoral.

L'application de tels principes, au regard de la littérature sur le projet participatif (Debarbieux et Lardon, 2003; Breton, 2009), suppose qu'une telle gestion ne peut se faire qu'au prix d'une concertation au sein des secteurs et entre les secteurs, et d'une sensibilisation très forte des professionnels aux questions de protection et de conservation. Elle repose également sur un principe de bénéfice réciproque, c'est-à-dire d'un développement économique accompagnant les restrictions dues à la conservation.

\section{La réalité de la pêche en Martinique : deux visions opposées}

La pêche professionnelle en Martinique pèse peu dans l'économie de l'île, elle ne représente que $0,16 \%$ du PIB total de l'île et $6 \%$ de l'emploi de l'économie bleue (Rostaing et al., 2016). Comparé aux secteurs du transport, du tourisme ou des activités sportives et de loisirs, son poids demeure faible. Toutefois, elle constitue un secteur structurant du point de vue social et spatial. Le nombre de marins professionnels est estimé à 1407 pour 1233 navires (Rostaing et al., 2016). Ces derniers sont de taille réduite (en moyenne $7,2 \mathrm{~m}$ et un équipage de 1,7 personne). Les effectifs et le nombre de navires sont relativement stables depuis les années 1990, mais les marins comme le parc de bateaux vieillissent avec plus de la moitié des marins ayant plus de 45 ans (Reynal et al., 2016). Les chiffres sont d'ailleurs variables d'une source à l'autre, y compris pour des données émises par des institutions gestionnaires (Rostaing, 2014; Vaillant, 2016). L'aquaculture est peu développée et se limite à une pratique artisanale dans des bacs en mer de l'ordre de $200 \mathrm{~m}^{2}$, très proches des côtes. À ce secteur professionnel, il est nécessaire d'ajouter la pêche dite de loisir dont il est difficile d'évaluer le poids, mais qui concerne aussi bien des populations de l'île que des touristes, lesquels peuvent exercer une pression importante sur les ressources : une étude BVA/Ifremer de 2005 estime à près de 4300 le nombre de chasseurs en plongée, la Fédération française d'études et de sports sous-marins (FFESSM) n'en recensant que 1723 (Rostaing et al., 2016). Dès lors, les institutions publiques sont confrontées à un double enjeu, diversement apprécié par les acteurs : la « modernisation » de la pêche professionnelle et le contrôle de la pêche de loisir.

Les rapports des institutions de la mer (Rostaing, 2014 ; Reynal et al., 2016; Vaillant, 2016) et les entretiens que nous avons réalisés auprès des institutions publiques insistent sur l'existence d'une crise de la pêche, et sur la nécessaire restructuration du secteur professionnel; 
l'argument principal étant le vieillissement du parc et des techniques (qui sont restées relativement traditionnelles). Par ailleurs, la pêche détient une mauvaise image auprès des institutions, celle d'un secteur associé à la pauvreté et déprédatrice du milieu naturel.

«La pêche en Martinique c'est 1080 marins pêcheurs professionnels en activité dont une majorité a plus de 50 ans. Ils ne seront pas tous en mesure de toucher une retraite décente. Un grand nombre de pêcheurs retraités continuent à s'enrôler et à pêcher. Des techniques de pêche non sélectives (casier, filet trémail, senne), néfastes pour la ressource halieutique et les habitats, sont encore largement utilisées. Ces pratiques aboutissent à une surexploitation du plateau insulaire et la ressource côtière est épuisée. Il est indispensable d'adapter les navires et les engins à de nouvelles méthodes de pêche plus sélectives et de mettre en place des zones de protection de la ressource halieutique. L'orientation professionnelle des pêcheurs actifs vers d'autres métiers de la mer (pescatourisme) peut être une source de revenus complémentaires. L'accompagnement social des pêcheurs retraités pourrait permettre de stopper leurs impacts négatifs sur la ressource et les milieux ${ }^{17}$.»

Ce discours, unanime au sein des institutions, fut répété à plusieurs reprises lors des réunions de construction du parc.

Au contraire, comme le révèlent les entretiens, les représentations des pêcheurs ${ }^{18}$ sont différentes. Ces derniers jugent le secteur plutôt dynamique et ne l'associent nullement à la pauvreté : "C'est un métier dur; il faut aller de plus en plus loin; mais on vit bien » (entrevue avec un pêcheur du Carbet). Les personnels de mairie interrogés indiquent que la plupart des pêcheurs et des matelots sont propriétaires de leur maison et de leur voiture, et qu'une grande partie ne vit que de la pêche. Les associations locales de pêcheurs fonctionnent bien et réussissent à capter des fonds pour l'installation d'infrastructures parfois importantes (frigorifiques, points de vente, pontons, etc.). Toutefois, l'organisation syndicale est un sujet de controverses, les pêcheurs souhaitant garder leur indépendance vis-à-vis des structures et du marché, ce qui tend à confirmer des constats établis de longue date (Dubost, 1996; 2002). Les marchés sont bien structurés ; la vente est assurée par les pêcheurs eux-mêmes ou leurs proches, directement aux professionnels (par téléphone avant débarquement) et au public sur les quais.

Les pêcheurs ne comprennent pas les nombreuses accusations des institutions portant sur leur rôle dans la dégradation des ressources, sur l'importance de la

\footnotetext{
${ }^{17}$ Synthèse patrimoine naturel, culturel et usages du 2 octobre 2014, document de deux pages distribué en séance, réalisé par la mission d'étude pour la création d'un parc naturel marin en Martinique.

${ }^{18}$ Enquête réalisée essentiellement à Case-Pilote, Bellefontaine, Le Carbet, Saint-Pierre et le Prêcheur.
}

pauvreté, sur leur conservatisme, etc., générant un sentiment de malaise qui tend à bloquer fréquemment les discussions. Certes, les pêcheurs reconnaissent qu'un travail de régulation de la ressource doit être entrepris; mais ils rejettent les accusations de prédateurs tout en mettant le doigt sur les nombreux problèmes engendrés par les autres secteurs : l'interdiction de la pêche de plateau sur $30 \%$ de la côte du fait de la contamination au chlordécone, mais aussi des cantonnements de pêche ; la croissance d'un secteur touristique polluant pour le milieu maritime (nuisances sonores causées par les scooters de mer, occupation des bateaux de plaisance, etc.); les nuisances du grand port maritime; la pêche illégale des grands chalutiers japonais; la pisciculture trop proche des côtes ; des problèmes liés aux espèces envahissantes comme la sargasse ou le poisson lion, etc. ; autant de thématiques et de questions non réglées ou prises en compte par la mission du parc dans ses discussions. Par ailleurs, les tentatives de reconversion de certains pêcheurs dans l'écotourisme (promenade en mer et observation des mammifères marins) sont loin d'être concluantes: elles créent des différences de statut dans l'usage des ressources et provoquent des conflits entre pêcheurs appartenant à une même association («Alors que les autres ils vont voir les dauphins, et ils gagnent 60 euros par personne; ils ont signé une charte pour bloquer les autres; ils sont 24 bateaux pour aller voir les dauphins; et moi je ne peux pas m'approcher, je dois rester à $300 \mathrm{~m}$; je ne peux même pas montrer les dauphins à ma famille... » (Entrevue avec un pêcheur de Case-Pilote).

On constate aussi de nombreux conflits d'usages: l'occupation de la plage par des cabanons et des ateliers, la pratique de la senne sur la plage, la présence permanente des pêcheurs et de leurs embarcations sur les plages sont autant d'infrastructures entrant en concurrence avec un tourisme normalisé nécessitant des réglementations. Ce sont également des éléments qui freinent la tendance à l'implantation d'un tourisme de luxe dans cette île des Caraïbes, très orientée vers la modernité et l'écologie (Dehoorne et Tafani, 2012). C'est dans ce contexte d'incompréhension réciproque que démarre le processus de concertation du PNMM; un processus dans lequel les pêcheurs sont loin d'être un allié «naturel». Leur participation et l'implication de leurs organisations (associations de producteurs) seront extrêmement limitées, comme le montre la figure $3: 25$ personnes issues $d u$ secteur de la pêche professionnelle seront présentes aux réunions durant les trois années sur 533 présences au total dans tout le processus; seules 6 participeront aux réunions de construction, n'effectuant qu'une dizaine d'interventions dans les débats (Fig. 4).

\section{Une stratégie d'auto-exclusion?}

Cette stratégie de non-participation fut volontaire d'après les entretiens effectués auprès des pêcheurs, à tel 


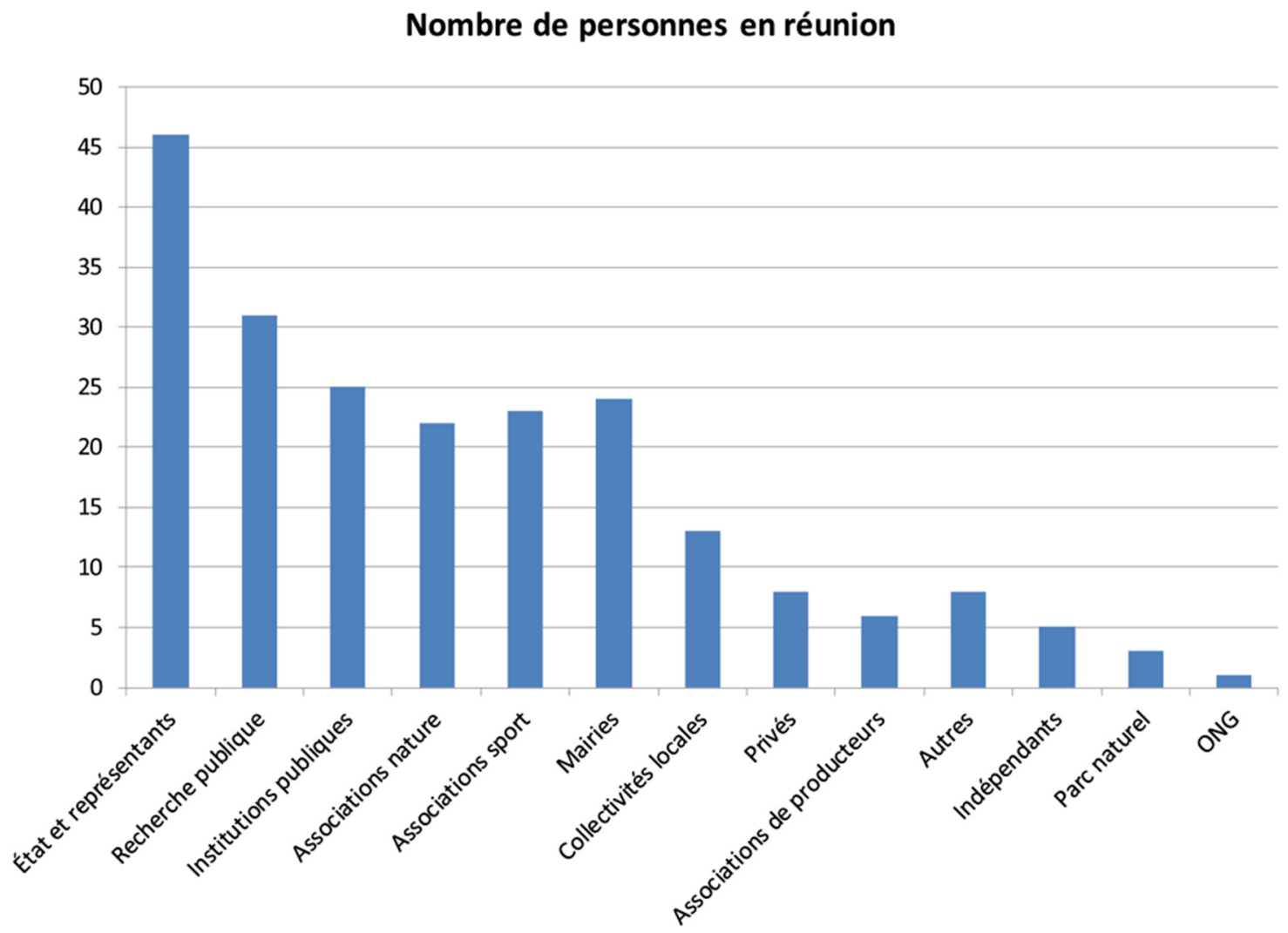

Fig. 3. Nombre de personnes ayant assisté aux réunions sur invitation de la mission du parc $(2013,2015)$ [source : feuilles de présence fournie par la mission].

point qu'il est permis de parler d'une pratique d'autoexclusion justifiée par plusieurs arguments.

Le principal argument résulte d'une perception de la participation comme d'un processus vertical, en provenance de l'État, faisant abstraction des principaux acteurs que sont les pêcheurs. Leur perception est celle d'un « divorce des savoirs » valorisant les savoirs incarnés par des experts au détriment de ceux développés localement et du patrimoine social. Les pêcheurs ou leurs représentants ne seront jamais conviés à exposer leur perception $\mathrm{du}$ milieu marin. Cent dix institutions, associations et individus furent invités par le préfet pour la première réunion du comité de concertation, mais seulement une association de pêche professionnelle (le comité régional des pêches maritimes et des élevages marins de Martinique - CRPMEM); les rares pêcheurs présents s'inviteront eux-mêmes, puis s'abstiendront de venir par la suite (selon l'analyse des feuilles de présence).

«Parce que chaque fois que quelque chose se fait à la Martinique, au départ ça va bien, après quelques jours les pêcheurs sont mis de côté, c'est pourquoi moi, là je parle en tant que syndicat, je ne suis pas d'accord sur cette position, je l'avais déjà dit au Lamentin que c'est trop long, il faudra faire un raccourci. Et si ce n'est pas le raccourci... parce que je suis en train de préparer un courrier pour faire savoir au préfet que trop de décisions sont prises dans le dos des marins pêcheurs et qu'ils ne sont pas respectés. Merci. » (A. L., marin-pêcheur de Sainte-Luce, réunion du 23/04/2015).

La seconde raison est l'absence de hiérarchisation des priorités des actions du parc: la déprédation reprochée aux pêcheurs est placée au même niveau que la pollution engendrée par le chlordécone, la croissance des sorties touristiques en mer ou la déprédation de sites d'oiseaux protégés, sans tenir compte de l'intérêt social ou économique. L'impératif de conservation n'est pas compris dans la mesure où les secteurs économiques polluants ne sont pas, eux, remis en cause. « Je voulais juste préciser que je ne crois pas que ce soit le pêcheur l'ennemi, c'est certainement la contamination, c'est certainement l'ensemble des produits déversés depuis très longtemps. Depuis le temps de la colonisation il y a eu des transports de matière, il y a des métaux lourds dans toutes les baies de la Martinique, toutes, du Nord au Sud.» (A.C. P., pêcheur de loisir et commissaire de l'enquête publique, réunion du 17/06/2014). «Sé pa péchè ki polié lan mè» (ce ne sont pas les pêcheurs qui ont pollué la mer), rappelle M. A., la présidente du syndicat indépendant des marins pêcheurs lors d'une manifestation à Fort-de-France ${ }^{19}$.

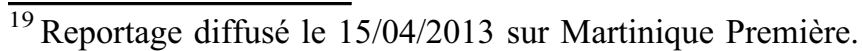




\section{Nombre d'interventions}

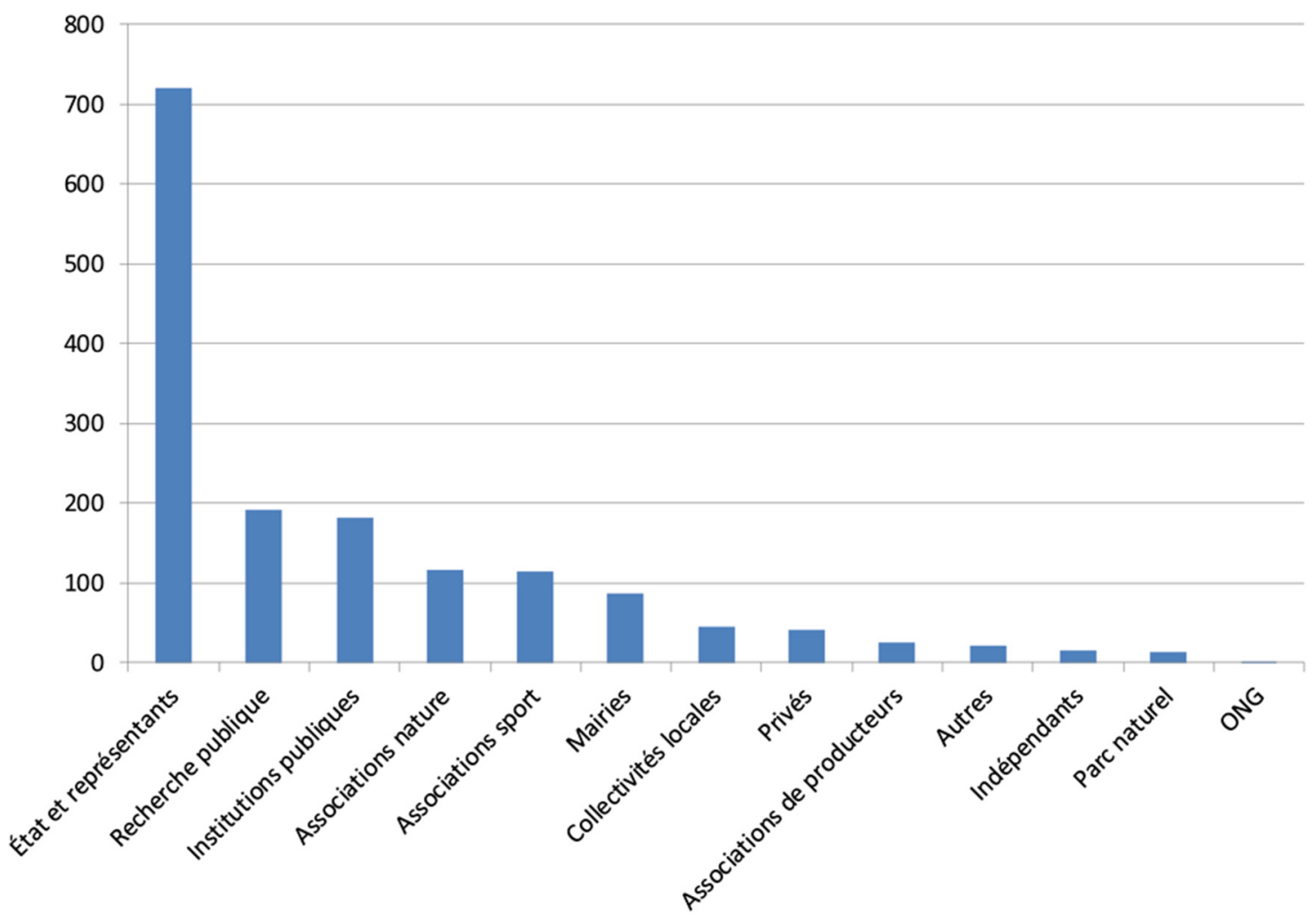

Fig. 4. Nombre d'interventions dans les débats selon le type d'acteur (source : analyse des verbatim des réunions ayant eu lieu entre 2013 et 2015, fournis par la mission du parc).

Une autre raison, plus technique, est associée à un sentiment d'exclusion face à une organisation et une assistance très formelles. Les dates et les heures de réunions, leur organisation dans un amphithéâtre peuplé de «cols blancs porteurs de cravates » n'ont pas favorisé la participation de certains secteurs productifs. Cet aspect a été plusieurs fois évoqué en réunion par des participants, mais les pratiques n'ont pas changé.

« Je suis inquiet de voir que les pêcheurs ne sont pas là. Cette absence est inquiétante. Elle est inquiétante d'autant plus qu'en général, les petits artisans sont le maillon faible de ce genre de réunion parce qu'ils doivent donner une journée de travail pour y venir. Ils ne sont pas forcément très à l'aise dans ce type de réunion face à des gens qui passent leur vie à lire des documents et à approfondir leur sujet. » (L. R., Ifremer, réunion du 02/ 10/2014).

Enfin, l'auto-exclusion découle de l'utilisation de méthodes qui tendent à la favoriser. En herméneutique, il n'y a ni vérité ni mensonge, mais différentes interprétations du message ou du discours (Ricœur, 1969). Dans le cadre d'une concertation, un même thème peut donner lieu à diverses interprétations, et beaucoup de doute subsistera sur les intentions de l'émetteur du message lorsqu'il est engagé dans une démarche de persuasion. La discussion rémanente sur la superposition des compétences des collectivités et celles de l'État est un exemple significatif à cet égard; il en est de même du débat sur l'interdiction ou la limitation de la pêche opposant les associations locales, le comité régional de pêche et l'État. Les postures adoptées se fondent alors sur l'exclusion de l'autre et le refus du dialogue; face à la centralité incarnée par l'État et les scientifiques, certains acteurs sortiront du processus avec un sentiment de doute et de refus (voir la déclaration du président du comité des pêches durant le forum Bòdlanmè en 2015 [Agence des 50 pas géométriques, 2015]). Plusieurs acteurs regretteront cette absence (inquiétante selon l'Ifremer) des pêcheurs, mais sans que ces derniers soient pour autant davantage sollicités, au moyen d'une réunion sectorielle par exemple. «On peut rajouter ce que l'on veut, écrire ce que l'on veut, mais ce sera toujours, semble-t-il, sans les acteurs. Vous parlez des pêcheurs, mais sans les pêcheurs. Les trois syndicats de pêcheurs n'ont jamais été invités aux réunions. Ils sont à part du comité des pêches, et de plus le comité n'est que consultatif pour les pêcheurs ${ }^{20}$.» Une opportunité a sans doute été perdue

\footnotetext{
${ }^{20}$ Réunion Construction Fort-de-France -2 octobre 2014, intervention de l'association des marins pêcheurs du Prêcheur appuyée par plusieurs associations et des mairies.
} 
d'offrir aux pêcheurs un vrai outil de gestion ${ }^{21}$, dans le sens où le propose Dubost, 2002: "Tout projet de développement doit s'inscrire dans l'univers social des pêcheurs pour, d'abord, trouver une légitimité, ensuite être réalisé par les pêcheurs eux-mêmes » (p. 139). Le constat est finalement préoccupant pour la suite de ce parc, puisque parmi les pêcheurs interrogés en 2017, on peut distinguer trois catégories de comportement qui influent sur leur perception collective et les possibilités d'une gestion collective concertée: ceux qui ont une méconnaissance totale du processus de concertation et de création du parc marin ou qui le confondent avec d'autres aires protégées (souvent celle du Prêcheur): en général de vieux pêcheurs isolés ou travaillant dans un milieu familial, peu reliés aux associations officielles; ceux qui récusent (sans les connaître vraiment) les interdictions supplémentaires accompagnant la création du parc et/ou condamnent le manque de concertation ou le mépris des institutions dans ce processus : essentiellement de petits pêcheurs (yoles) bien insérés dans les associations locales; ceux qui sont indifférents et pensent que le parc ne les affectera pas, car c'est un «truc» de l'État: des pêcheurs pratiquant sur de gros bateaux, pêchant au large, parfois jusqu'aux côtes du Venezuela. Dans tous les cas, le processus de concertation fut le révélateur d'une triple fragmentation des acteurs: entre secteurs (selon le type de pêche), interne au secteur (par rapport aux syndicats et au secteur du pescatourisme) et territoriale (Nord-Sud et Est-Ouest, en fonction des zones de pêche et de leur relation aux autres activités). Très peu sont informés et sont prêts à changer leurs pratiques. Il est à noter que, finalement, c'est le président d'une association de pêche de loisir, très critique sur le processus de concertation, qui prendra en main l'enquête publique, laquelle se terminera par un avis négatif. De même, c'est le président du comité de pêche, opposant à la création du parc, qui sera élu président du comité de gestion du même parc. Ces tensions sont un défi évident dans le fonctionnement de ce comité de gestion.

\section{Parc naturel marin de Martinique et associations : un rendez-vous manqué?}

\section{L'enjeu de l'acceptation à travers la participation}

Durant toutes les périodes préliminaires à la mise en place du PNMM, de l'analyse stratégique régionale de

\footnotetext{
${ }^{21}$ «Alors que la pêche traditionnelle fait partie des objectifs, on ne comprend pas pourquoi on n'a pas saisi l'opportunité d'offrir aux pêcheurs un outil de gestion, alors qu'ils le demandent » (Intervention de l'Office de l'eau lors de la réunion de restitution -23 avril 2015 sur le périmètre).
}

Martinique (ASRM), réalisée entre 2009 et 2010, à la mission de concertation qui débute fin 2013, le milieu associatif et scientifique martiniquais a été amplement sollicité, en particulier pour documenter le volet connaissance du projet. Sa contribution à l'élaboration de l'état des lieux/diagnostic est majeure. Elle porte sur l'état des connaissances floristiques, faunistiques et écosystémiques du littoral et de la mer ainsi que sur l'identification des enjeux autour du patrimoine naturel (activités anthropiques, pollutions, déséquilibres écosystémiques). Par ailleurs, par intérêt scientifique, mais aussi politique et économique, les associations de protection de la nature s'avèrent des alliés objectifs du PNMM. Au début de la phase de concertation, elles sont de nouveau sollicitées sous forme de contributions expertes lors de réunions de concertation. Puis peu à peu, elles disparaissent du débat et des réunions de concertation.

Leur perception de ce parcours a fait l'objet d'entretiens avec les associations de protection de la nature et d'usagers de l'environnement, ce qui nous a permis de les classer en fonction de leur stratégie vis-à-vis du parc:

- Associations de protection de l'environnement à capacités d'expertise scientifique (Carbet des sciences, Carouge, Observatoire du milieu marin martiniquais [OMMM], Société Étude protection et aménagement de la nature à la Martinique [SEPANMAR]) ;

- Associations de sensibilisation à l'environnement développant un savoir-faire pédagogique (AssoMer, La Case/Chambres régionales de l'économie sociale et solidaire [CRESS], GRAINES, Entreprise \& Environnement, Les Jardins de la Mer);

- Associations de protection de l'environnement à capacités de mobilisation citoyenne (Association pour la protection de la nature et de l'environnement [APNE], Association pour la sauvegarde du patrimoine martiniquais [ASSAUPAMAR]);

- Associations d'usage du milieu marin et littoral (Basile/Association des maîtres-nageurs sauveteurs de Martinique [AMNSM]), Comité régional des activités et sports subaquatiques [COMASSUB], Fédération martiniquaise de la pêche plaisancière [FMPP], OCEANA, CRESSMA, Surf Rider [pour l'essentiel des clubs de plongée]).

Il n'est guère besoin d'insister sur les associations d'usage du milieu. Leur posture vis-à-vis du PNMM peut être qualifiée d'attentisme bienveillant. Elles se placent en observatrices du processus et en attendent beaucoup en matière de protection de l'environnement et de restauration des écosystèmes, et surtout en bénéficient pour leurs activités. Une association, la FMPP, échappe à cette attitude de non-participation cordiale en étant présente aux réunions de concertation. Les associations «citoyennes» APNE et ASSAUPAMAR diffèrent en 
tout point l'une de l'autre, sur la fin comme sur les moyens. La première concentre son objet sur la protection de l'environnement. Son adversaire est l'acteur qui, par ses activités, dégrade les écosystèmes et la biodiversité. La puissance publique est un arbitre à qui l'on s'adressera pour faire respecter la législation. L'ASSAUPAMAR, elle, développe des objectifs multiples: droits de l'homme, santé, écologie, dans une approche quasi systémique des problèmes de société et d'environnement. Son adversaire est multiforme: tout acteur qui contrevient aux droits humains et à ceux de la nature, y compris la puissance publique. La tournure politique mise dans chacune de ses actions fait de l'ASSAUPAMAR un partenaire à part au sein du projet PNMM, à la fois incontournable et critique. Enfin les associations expertes ou pédagogiques sont souvent l'émanation d'une personne ou de groupes restreints qui s'en servent comme tremplin pour valoriser leurs expertises ou savoir-faire. Dans un premier temps, elles accueillent favorablement le projet PNMM, car elles espèrent que celui-ci donnera à leur développement de nouveaux moyens institutionnels et matériels. La réalité est tout autre: le projet de parc les fragilise, les obligeant à réduire la voilure, à chercher ailleurs les moyens de leur survie et à mettre en sourdine leur association, en attendant d'hypothétiques jours meilleurs.

Face à la diversité des associations locales actrices de la mer et du littoral, qui s'accordent sur les fins à défaut de s'accorder sur les moyens, l'État promoteur du projet de parc poursuit des objectifs qui visent avant tout à satisfaire les engagements internationaux pris par la France pour répondre aux injonctions des paradigmes qui sous-tendent la création des aires marines protégées: pressions anthropiques et climatiques (Bonnin et al., 2015); modèle écosystémique réticulé (David et al., 2015); gestion intégrée (Cacqueray et al., 2015). La mission du parc, intermédiaire dans ce dialogue, a reconnu, lors d'un entretien, qu'il pouvait y avoir un hiatus entre les objectifs poursuivis par l'État, notamment à travers ses engagements internationaux, et les aspirations locales à la protection et au développement.

\section{Éléments de convergence}

Dans le cadre du processus de création du PNMM, il ne manquait pourtant pas d'éléments de convergence pour mettre en perspective les objectifs de l'État et les aspirations locales portées par les associations ainsi que des outils pour les réaliser. Le PNMM est un projet d'essence participative. En effet, l'outil parc naturel marin a été créé en 2006 à la suite des seize années de vicissitudes et de conflits autour du projet de parc de la mer d'Iroise (Van Tilbeurgh et Charlier-Kerbiguet, 2009). Les trois années de concertation qui découlent de cette expérience en mer d'Iroise semblent une ressource temporelle suffisante pour débattre des conflits d'usage et de pouvoir, et parvenir à un consensus. Il y a notamment des promesses de création d'un conseil de gestion ayant des prérogatives jugées intéressantes par certains acteurs associatifs (notamment les avis conformes), car il fait la part belle aux acteurs locaux. Enfin l'État promoteur présente une garantie de moyens qui comble les associations détentrices d'expertises et de savoir-faire sur le milieu marin et littoral. Le parc ambitionne la protection et la restauration du milieu marin, présenté comme un patrimoine naturel à valoriser, et le développement durable des activités marines ${ }^{22}$, double ambition à laquelle souscrit l'ensemble du milieu associatif. Par ailleurs, le milieu associatif jouit d'une reconnaissance de la part des services de l'État avec lesquels une collaboration de longue date s'est instaurée. La direction de l'Environnement, de l'Aménagement et $\mathrm{du}$ Logement (DEAL) possède un répertoire des associations et utilise leurs services en tant qu'opérateur. Nous avons vu que les promoteurs du parc ont largement puisé dans leur expertise et leur savoir-faire pour documenter et avancer leur projet.

\section{Les germes d'un divorce}

Mais ce qui avait commencé comme une idylle entre l'État promoteur du projet et les associations s'est progressivement transformé en divorce, ces dernières prenant leur distance en cours de concertation. Pour la plupart, elles estiment ne pas avoir été entendues. Mais les causes de ce rendez-vous manqué sont bien plus complexes qu'une simple communication déficiente. La mission d'études en charge de la concertation a fait face à des contraintes horizontales et verticales qui l'ont bridée dans ses relations avec les associations.

Parmi les contraintes horizontales, citons le hiatus entre l'État décisionnaire et l'État gestionnaire. Le premier, d'essence politique, tire la légitimité de ses décisions de son mandat électoral, le second, d'essence administrative, se vit comme un tampon chargé de les appliquer localement. L'analyse chronologique de la documentation de la mission va montrer que l'État gestionnaire, à travers ses différentes composantes (DEAL, direction de la Mer), va peu à peu se substituer aux associations dans l'établissement des bases d'informations.

La verticalité de la décision politique gêne aussi la mission d'études, rendant la procédure de concertation quelque peu rigide et difficilement adaptable au contexte local. Le personnel de la mission prétend avoir carte blanche sur la façon dont il mène cette concertation, mais le peu d'engouement de l'AAMP pour la connaissance

\footnotetext{
${ }^{22}$ Selon l'Agence française de la biodiversité qui gère maintenant les aires marines protégées.
} 
des sociétés locales et son appétence quasi exclusive pour les données environnementales n'ont pas permis à la mission de mandater les experts locaux afin de mieux connaître la situation sociale, culturelle et productive locale. Nos entretiens avec la mission du parc et l'examen des documents de diagnostic montrent en effet un net déséquilibre entre la précision des diagnostics environnementaux et la généralité des données socioéconomiques.

Enfin, la question politique et territoriale s'exprime de diverses façons. À l'instar des sociétés insulaires très territorialisées et défiantes comme l'archipel de Molène (Chlous-Ducharme, 2004), la FMPP va défendre les intérêts des pêcheurs plaisanciers selon une pratique gestionnaire de l'environnement littoral et marin, revendiquée comme traditionnelle et territorialisée. Les associations «citoyennes» comme l'ASSAUPAMAR ont participé à la concertation et revendiquent une place dans le conseil de gestion du futur parc. Il ne s'agit pas d'une adhésion au sens propre mais d'être présent pour faire valoir ses vues et « surveiller» les agissements de l'État. La question du rapport colonial est certes très peu exprimée et souvent en filigrane; mais elle n'est pas absente des discours de certaines associations solidement implantées ou au contraire fragiles et en veilleuse.

En raison de leur dépendance vis-à-vis de la puissance publique, les associations «expertes » formulent des critiques plus feutrées. Elles reconnaissent la légitimité des stratégies nationales appuyées sur le PNMM et vont jusqu'à encourager la ministre dans sa quête d'un poste international ${ }^{23}$. Les critiques concernent la non-prise en compte de leur compétence et de leurs conseils, leur mise à l'écart de toute opérationnalité présente et future dans le PNMM. Elles hésitent entre une stratégie d'entrisme dans le futur conseil de gestion et rester des opérateurs potentiels pour les projets qui en émaneront.

\section{Un rendez-vous manqué}

Au-delà d'une sorte de rendez-vous manqué entre les promoteurs étatiques du projet de PNMM et les associations locales de protection de la nature et d'usage du milieu naturel, ces relations sont symptomatiques d'une difficulté à faire de la concertation un espace de vrais débats où les conflits d'usages et de pouvoir pourraient être exposés et permettre d'élaborer un consensus. La mission d'études fut finalement bridée par une procédure de concertation normée qui exclut l'innovation. Elle a été aussi tributaire des administrateurs et des élus locaux qui

\footnotetext{
${ }^{23}$ Référence à la candidature de la ministre de l'Environnement au poste de présidente du Programme des Nations unies pour le développement (PNUD).
}

semblent se désengager progressivement du financement des opérateurs associatifs. Depuis l'expérience de la mer d'Iroise, la protection intrinsèque du milieu n'est plus, en soi, un paradigme acceptable. La connexion milieu-société se fait par le biais de la patrimonialisation de la nature et la mise en exergue de l'idée de transmission de ressources matérielles et immatérielles (Van Tilbeurgh et CharlierKerbiguet, 2009; Claeys et al., 2016). Il n'est pas certain que cela suffise à susciter l'adhésion des acteurs locaux. Enfin, les associations sont perçues comme inoffensives pour le projet de parc car en tant qu'opératrices, elles sont dépendantes de l'État gestionnaire et des collectivités territoriales pour financer leurs activités. Le nombre de places allouées au sein du conseil de gestion est, de ce point de vue, significatif: 5 représentants pour les associations de protection sur 51 membres. Le glissement de la position des associations du statut d'alliés objectifs du PNMM à celui d'opposants n'est pas de bon augure à l'heure où il faudra concrétiser ce projet dans les faits à travers le conseil de gestion du parc.

\section{Discussion}

\section{Les limites du processus participatif}

Les limites du processus consultatif ressortant de cette étude interrogent sur le contenu et la portée du principe de participation tel qu'il est posé par le droit français. Ce principe, consacré d'abord par la loi ${ }^{24}$ puis à l'échelon constitutionnel ${ }^{25}$, reconnaît le droit de toute personne d'être informée des projets de décisions publiques ayant une incidence sur l'environnement et de disposer de conditions lui permettant de formuler ses observations. Il implique parallèlement l'obligation pour l'autorité publique compétente d'offrir au public des conditions favorables à sa participation et de prendre en compte les observations formulées ${ }^{26}$. Il s'agit d'un droit procédural (Pissaloux, 2011) qui prévoit la mise en œuvre de moyens, sans toutefois garantir l'atteinte du but d'effectivité recherché. Car la participation effective,

\footnotetext{
${ }^{24}$ Loi n ${ }^{\circ} 95-101$ du 2 février 1995 relative au renforcement de la protection de l'environnement (loi Barnier), Journal officiel, 29, 3 février 1995, 1840.

${ }^{25}$ Article 7 de la Charte de l'environnement adossée à la Constitution française par la loi constitutionnelle $n^{\circ} 2005-205$ $\mathrm{du} 1^{\text {er }}$ mars 2005 relative à la Charte de l'environnement, Journal officiel, 51, 2 mars 2005, 3697, texte $\mathrm{n}^{\mathrm{o}} 2$.

26 «Le principe de participation en vertu duquel toute personne est informée des projets de décisions publiques ayant une incidence sur l'environnement dans des conditions lui permettant de formuler ses observations, qui sont prises en considération par l'autorité compétente ; (Article L. 110-1 du Code de l'environnement, II. $5^{\circ}$ ).
} 
bien qu'évoquée dans les différents textes de droit ${ }^{27}$ sous la forme d'un objectif visé, ne constitue pas une exigence juridique de résultat. Elle repose sur la volonté du public de se saisir de ce droit.

La mise en œuvre du principe de participation ne consiste pas en un processus de codécision. D'une façon générale, les procédures consultatives souffrent d'une crise de confiance de la part du public et sont visées par de nombreuses critiques quant à leur place et leur utilité dans le processus décisionnel. Elles apparaissent plus informatives que consultatives, voire persuasives (Blondiaux, 2008; Blatrix, 2009; Claeys-Mekdade et al., 2009). L'autorité décisionnelle garde le dernier mot. C'est pourquoi souvent demeure dans l'esprit du public le sentiment que le projet est ficelé d'avance. Et en Martinique, c'est l'idée que Paris, l'État, veut imposer son dispositif.

Les observations formulées par le public, bien que devant être «prises en compte», n'ont pas d'incidences substantielles sur le projet. De même, le défaut de participation effective n'affecte pas le processus décisionnel. La non-participation peut d'ailleurs être perçue comme une forme de participation. L'autorité décisionnelle n'est également pas liée par l'avis rendu par les commissaires-enquêteurs à l'issue de l'enquête publique. Le désintérêt du public pourrait donc s'expliquer par le sentiment que tout est déjà joué d'avance, la consultation n'ayant aucun impact significatif sur la décision finale, et le processus participatif, véritable «trompe-l'œil», « simulacre de démocratie » (Blondiaux, 2008; Blatrix, 2009), lui apparaissant comme inutile. Ainsi le cas martiniquais s'ajouterait-il aux nombreux exemples témoignant du manque de crédibilité dont souffre la démocratie participative auprès des populations. En effet, malgré un avis défavorable des commissaires-enquêteurs, les acteurs opposés au PNM à la Martinique se trouvent confrontés à l'affirmation d'une doctrine de la préservation du patrimoine naturel consacrée par la promulgation de la loi $^{28}$ pour la reconquête de la biodiversité, de la nature et des paysages.

\footnotetext{
${ }^{27}$ À titre d'exemples : articles 6 et 8 de la Convention d'Aarhus du 25 juin 1998 sur l'accès à l'information, la participation du public au processus décisionnel et l'accès à la justice en matière d'environnement, Journal officiel de l'Union européenne, L 124, 17 mai 2005; Directive 2003/35/CE du Parlement européen et du Conseil du 26 mai 2003 qui reprend les exigences de la Convention d'Aarhus, Journal officiel de l'Union européenne, L 156, 25 juin 2003 ; l'article L. 120-1 du Code de l'environnement. Ces textes évoquent ce en quoi consiste la participation effective, ou les dispositions nécessaires «pour permettre une participation effective» ou pour permettre au public «de participer effectivement».

${ }^{28}$ Loi n $^{\circ} 2016-1087$ du 8 août 2016 pour la reconquête de la biodiversité, de la nature et des paysages, Journal officiel, 184, 9 août 2016 , texte $\mathrm{n}^{\mathrm{o}} 2$.
}

Les tensions sociales qui se sont cristallisées autour de l'équipe en charge de la concertation peuvent être interprétées comme des résultantes de frustrations occasionnées par l'impossibilité de gérer efficacement les controverses environnementales, par exemple celles autour des invasions de sargasses, sur une durée de trois ans. À la Martinique, la mise en œuvre de l'action collective est contrainte par des situations où le principe de précaution est une réponse récurrente à une forme d'exacerbation d'incertitudes des savoirs et de risques environnementaux (Lalubie et al., 2015). L'équipe de l'AFB en charge de la concertation préalable à la création du PNM de Martinique a préféré résoudre les potentiels conflits par une stratégie d'évitement. Or la «réhabilitation du conflit» (Bobbio et Melé, 2015) est un prérequis indispensable à la participation dans le cadre de la gestion intégrée des zones côtières (Billé, 2006).

\section{Au-delà du participatif, quel avenir pour le conseil de gestion?}

Au-delà des critiques dont elle fait l'objet, la participation est considérée comme un moyen d'améliorer la qualité de la décision publique et de contribuer à sa légitimité démocratique ${ }^{29}$. Ainsi deux nouvelles démarches, l'une en rapport avec le fonctionnement $\mathrm{du}$ conseil de gestion, l'autre en direction du grand public, sont susceptibles d'en améliorer l'effectivité. Elles sont exposées ci-dessous.

Des modes de gestion basés sur la gouvernance environnementale se développent, encouragés notamment par la mouvance du développement durable. À cet égard, le conseil de gestion du PNM s'inscrit dans cette démarche de gouvernance. À l'instar de tous les conseils de gestion de parc marin, il s'agit d'un organe collaboratif et consultatif, composé de représentants des catégories d'acteurs concernés par le parc et au sein duquel la concertation et la négociation doivent occuper une place importante. Un tel dispositif semble présenter une forte ambition de concertation entre les acteurs impliqués dans le parc. Mais cela ne suffit pas à en préjuger l'efficience. Le fonctionnement du conseil devrait dépendre de l'aptitude des acteurs à se saisir de ce dispositif et à collaborer en mettant en place des dispositifs de régulation des conflits inhérents à toute organisation. Par ailleurs, pour en éviter le dévoiement, le Code de l'environnement charge l'Agence française pour la biodiversité de la gestion du parc ${ }^{30}$ et accorde au représentant de l'État les fonctions de commissaire du gouvernement de façon à exercer un contrôle sur le

\footnotetext{
${ }^{29}$ Article L.120-1 du Code de l'environnement.

${ }^{30}$ Article L.334-4 du Code de l'environnement.
} 
fonctionnement $\mathrm{du}$ conseil de gestion en cas de désaccord entre les parties ${ }^{31}$.

Plus généralement, des réflexions ont été entreprises, à la demande du gouvernement, afin d'améliorer le dialogue environnemental et le processus participatif (Richard, 2015). Des chercheurs et des experts ont évoqué l'idée d'engager auprès du public une démarche pédagogique en matière de participation, dans le cadre notamment de l'éducation à l'environnement. En outre, le ministère de l'Écologie et du Développement durable a publié en 2016 une charte de la participation du public. Ce texte est dépourvu de force contraignante: il relève davantage du droit souple (Zarka, 2016), mais a néanmoins une vertu pédagogique indéniable, car il pose les principes sur la base desquels sera organisée la concertation et permet de la sorte d'en informer le public (équivalence de traitement des points de vue, respect des savoirs et des expertises d'usage, tiers garant, etc.). Il a également un caractère novateur en ce qu'il encourage le porteur de projet à engager une démarche proactive (ce qui implique d'aller à la rencontre des publics concernés), une marge de manœuvre lui étant accordée dans le choix du degré d'implication souhaité du public dans le processus décisionnel, allant de la simple consultation à la codécision. Ce type d'outils est susceptible d'inspirer d'autres initiatives plus sectorielles et d'augmenter l'intérêt des populations pour le droit à la participation.

Le conseil de gestion du parc de Martinique tarde à se réunir, mais le suivi de la perception qu'en ont les acteurs sera important pour mettre en évidence d'éventuels processus d'appropriation.

\section{Conclusion}

Centrées sur le paradigme du développement durable, les aires marines protégées ont aussi des implications sociales sur le rapport de la société à l'environnement selon des modalités qui n'existaient pas forcément avant la création de ces aires. Intégrer ces nouvelles modalités ou pratiques du développement durable demande une certaine participation sociale dans les processus de décision (Santos, 2011). La conservation est un enjeu

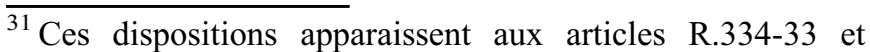
R.334-35 du Code de l'environnement. Les dispositions de l'article R.334-35 ont été modifiées par le décret n²016-1842 du 26 décembre 2016 relatif à l'Agence française pour la biodiversité, Journal officiel, 300, 27 décembre 2016, texte $\mathrm{n}^{\mathrm{O}} 3$. L'AFB est l'établissement public (créé par la loi n²0161087) qui assure désormais la gestion des PNM remplaçant l'AAAMP (article L.334-4 du Code de l'environnement).
}

universel et accepté par tous, difficilement critiquable comme objectif de développement, mais le processus participatif qui devait conduire à un projet approprié par tous n'a finalement révélé qu'une fragmentation des enjeux et des acteurs.

Au départ, la plupart des acteurs que nous avons étudiés (pêcheurs et associations) étaient plutôt perplexes, mais ouverts à la possibilité d'un projet d'aire marine. Toutefois la conduite même du processus les a convaincus que ce projet n'était qu'un jeu des institutions autour de réglementations et, par assimilation, d'interdictions. Cette fragmentation des acteurs et des enjeux s'est produite à partir de trois lacunes de la concertation : un effet «entonnoir» (réunions de plus en plus limitées aux seules institutions, sans prise en compte des avis énoncés lors des débats), une perte d'intérêt (stratégies divergentes et des prises de pouvoir de certaines associations), l'absence d'information (les pêcheurs peu informés, peu organisés, se sentent dépossédés de leur vie sociale, de leurs connaissances, voire de leurs traditions).

Par ailleurs, l'analyse de ce cas martiniquais a révélé, sous un prisme grossissant, les limites inhérentes au modèle français de débat public ainsi que l'influence du contexte local sur les stratégies des acteurs. Tout d'abord, la réalité martiniquaise est celle d'une société civile certes foisonnante, mais fragmentée et faiblement structurée, aboutissant mécaniquement à renforcer le poids des institutions étatiques et municipales dans le cadre des échanges. Il en résulte une captation de la parole par les représentants de ces institutions, érigés par la force des choses en professionnels d'une concertation reposant davantage, selon une tradition bien établie, sur l'idée d'une nécessaire explication de l'action des pouvoirs publics que sur des échanges constructifs (Le Quino, 2017). De nombreuses associations ont tenté d'investir le champ de ladite concertation, mais leur participation au fil des réunions s'est révélée intermittente alors que d'autres, souvent influentes, à l'instar du syndicat indépendant des marins pêcheurs et du comité citoyen du Sud, sont restées délibérément cantonnées dans une stratégie défensive annonçant sans doute des mobilisations a posteriori contre la mise en œuvre du PNMM.

À cela s'ajoute un deuxième facteur explicatif: la dissonance entre, d'un côté, un intérêt local fréquemment valorisé à partir de savoirs et de pratiques "profanes", voire d'affirmations identitaires et, de l'autre, des savoirs ou expertises «scientifiques» réputés imperméables à la culture locale parce que frappés d'altérité et portés par un État faiblement ancré dans la réalité du terrain.

Enfin, dans un contexte où persistent au sein du paysage politico-institutionnel, de manière confuse mais incontestable, des revendications en faveur d'une plus 
grande autonomie décisionnelle ${ }^{32}$ et de gestion de la société civile, des jeux de posture et de pouvoirs tendent inévitablement à se greffer sur les échanges ou servent à justifier le retrait de certains acteurs de la scène, notamment autour de la question éternellement débattue de la pertinence et de la légitimité des échelons de décision (Thirot et al., 2017).

Faut-il pour autant s'en tenir à ces constats quelque peu désabusés? Répondre à cette question revient à dépasser un paradoxe bien connu: d'un côté, la participation est appréhendée comme un horizon indépassable, alors qu'elle repose, de l'autre, sur des injonctions juridiques peu contraignantes pour le décideur et peu mobilisatrices pour les structures associatives réduites au statut peu enviable de participants entièrement privés du pouvoir de décision (Bouvier, 2007). Sans doute conviendrait-il, au-delà de la portée juridiquement limitée des dispositifs qui en constituent le substrat, d'en faire non pas un prétendu lieu de partage du pouvoir décisionnel, mais plutôt un espace d'échanges, de circulation des informations et de confrontation d'options apte à nourrir plus largement le débat public et à renforcer la légitimité des décisions. Dès lors, dans une perspective plus optimiste, des événements qui sont survenus en 2017, comme la mobilisation d'un syndicat indépendant de marins pêcheurs ${ }^{33}$ et la saisine du Conseil d'État par un maire opposé à la création du PNM de Martinique, annoncent l'émergence de rapports de force et de pouvoir. Dans le prolongement de la publication du décret, ledit syndicat s'est mobilisé, avec le soutien de l'association «Comité citoyen du sud», contre la création du parc naturel marin en dénonçant notamment son impact sur les ressources et les activités humaines. Il est intéressant de noter que L'Asso-Mer, une association de sensibilisation et de protection du milieu marin, a pris la défense du parc. Dans ces conditions, l'un des premiers enjeux du PNM de Martinique consistera donc à affirmer sa légitimité en régulant ces clivages.

\footnotetext{
${ }^{32}$ Toute l'histoire de la Martinique est rythmée par des revendications statutaires portées par les mouvements politiques, dont certains sont favorables à une plus large autonomie locale. Aujourd'hui, les forces ou les leaders politiques à la tête des institutions décentralisées (collectivité territoriale de Martinique, établissements publics de coopération intercommunale et la plupart des communes) reprennent à leur compte cette revendication, même si elle est exprimée de manière parfois confuse. Un autre élément complique la situation: ladite revendication est couplée, dans certains cas, à une défense de l'identité ou du patrimoine culturel fortement valorisés localement (Daniel, 2002).

${ }^{33}$ Manifestation du 10 juillet 2017 sur le thème «Les marins pêcheurs n'ont pas été consultés », en opposition à la création du parc marin. Voir l'article de France-Antilles Martinique du 30 juin 2017.
}

Finalement, on peut considérer que la vulnérabilité des littoraux, dans ses enjeux environnementaux, est accentuée par l'absence de projet territorial (dans le sens de la construction d'une vision partagée) et par une présence de l'État trop verticale. Le «forçage social » sur des approches dites intégrées et nécessitant l'acquisition de connaissances expertes, au nom d'une légitimité indiscutable, a définitivement montré ses limites, quels que soient les espaces concernés et les procédures (Spotorno, 2005; Billé, 2006; Dahou et Ould Cheikh, 2007; Weigel et al., 2007; Aubertin et Rodary, 2008; Lequin, 2009).

\section{Références}

AAMP (Agence des aires marines protégées), 2010. Analyse stratégique régionale Martinique. Enjeux et propositions de création d'aires marines protégées, Brest, AAMP.

Agence des 50 pas géométriques, 2015. Les aires marines protégées : un outil de réconciliation des usagers ?, verbatim du forum Bòdlanmè, 16-18 juin, Madiana, Brest, AAMP, www.aires-marines.fr/L-Agence/Organisation/ Parcs-naturels-marins/Parc-naturel-marin-de-Martinique/ Documentation/Verbatim-Forum-Bodlanme-2015-Lesaires-marines-protegees-un-outil-de-reconciliation-des-usa gers/.

Aubertin C., Rodary E. (Eds), 2008. Aires protégées, espaces durables?, Marseille, IRD Éditions.

Billé R., 2006. Gestion intégrée des zones côtières : quatre illusions bien ancrées, Vertigo, 7, 3, doi: 10.4000/ vertigo. 1555 .

Blatrix C., 2009. La démocratie participative en représentation, Sociétés contemporaines, 2, 74, 97-119, www.cairn. info/revue-societes-contemporaines-2009-2-page-97. htm.

Blondiaux L., 2008. Démocratie délibérative vs. démocratie agonistique? Le statut du conflit dans les théories et les pratiques de participation contemporaines, Raisons politiques, 30, 2, 131-147, www.cairn.info/revue-raisons-politi ques-2008-2-page-131.htm.

Bobbio L., Melé P., 2015. Introduction. Les relations paradoxales entre conflit et participation, Participations, 13, 3, 7-33, www.cairn.info/revue-participations-2015-3page-7.htm.

Bonnin M., Laë R., Behnassi M., 2015. Toujours plus d'aires marines protégées !, in Bonnin M., Laë R., Behnassi M. (Eds), Aires marines protégées ouest-africaines. Défis scientifiques et enjeux sociétaux, Marseille, IRD Éditions, 7-28.

Bouvier A., 2007. Démocratie délibérative, démocratie débattante, démocratie participative, Revue européenne des sciences sociales, 45, 1, 5-34, https://journals.openedi tion.org/ress/82.

Breton J.-M., 2009. Espaces et aires protégés. Gestion intégrée et gouvernance participative, Études caribéennes, 12, http:// journals.openedition.org/etudescaribeennes/3558. 
Cacqueray M. de, Rocle N., Meur-Férec C., Denis J., Henocque Y., David L., 2015. Atoumo. Vers une gestion intégrée de l'île de la Martinique et de son espace maritime. Trajectoires de gouvernance et adaptation aux changements passés, actuels et futurs, Brest/Saint-Denis (La Réunion), Université de Bretagne-Occidentale/LETG-Brest Géomer/DEAL de La Réunion/Ifremer, www-iuem.univbrest.fr/pops/attachments/607/Rapport_final_V4_062015office.pdf.

Chlous-Ducharme F., 2004. L'archipel de Molène et «l'Autre bord», Ethnologie française, 34, 1, 113-122, doi.org/ 10.3917/ethn.041.0113.

Claeys-Mekdade C., Leborgne M., Ballan E., 2009. Cadrer la procédure de concertation pour construire la confiance ? Arles, Marseille, Le Verdon, in Mermet L., Berlan-Darqué M. (Eds), Environnement. Décider autrement : nouvelles pratiques et nouveaux enjeux de la concertation, Paris, L'Harmattan, 97-116.

Claeys C., Hérat A., Barthélémy C., Deldrève C., 2016. Quand les Calanques deviennent Parc National : disputes autour de la définition et de la répartition des efforts environnementaux et urbains induits, Norois, 238-239, 1-2, 71-84, www. cairn.info/revue-norois-2016-1-page-71.htm.

Dahou T., Ould Cheikh A.W., 2007. L'autochtonie dans les aires marines protégées. Terrain de conflit en Mauritanie et au Sénégal, Politique africaine, 108, 4, 173-190, doi.org/ 10.3917/polaf.108.0173.

Daniel J., 2002. L'espace politique aux Antilles françaises, Ethnologie française, 32, 4, 589-600, doi.org/10.3917/ ethn.024.0589.

David G., Chabanet P., Lagabrielle E., Pennober G., Quod J.P., 2015. Les aires marines protégées face au changement climatique. De la résilience écosystémique à la résilience des territoires, in Bonnin M., Laë R., Behnassi M. (Eds), Aires marines protégées ouest-africaines. Défis scientifiques et enjeux sociétaux, Marseille, IRD Éditions, 67-80.

Debarbieux B., Lardon S., 2003. Les figures du projet territorial, La Tour-d'Aigues/Paris, l'Aube/Datar.

Dehoorne O., Tafani C., 2012. Le tourisme dans les environnements littoraux et insulaires: permanences, limites et perspectives, Études caribéennes, 19, http:// journals.openedition.org/etudescaribeennes/5774.

Dubost I., 1996. De soi aux autres.. Un parcours périlleux : la construction d'un territoire par les pêcheurs martiniquais. Thèse de doctorat en sciences sociales, Toulouse, Université Toulouse-Jean Jaurès.

Dubost I., 2002. Gestion du risque et de l'aléatoire par les pêcheurs martiniquais, in Blanchet G., Gobert B., Guérédrat J.-A. (Eds), La pêche aux Antilles. Martinique et Guadeloupe, Paris, IRD Éditions, 125-140.

Féral F., 2011. L'extension récente de la taille des aires marines protégées : une progression des surfaces inversement proportionnelle à leur normativité, Vertigo, hors-série, 9, doi: $10.4000 /$ vertigo. 10998 .

Godet M., 1997. Manuel de prospective stratégique, Paris, Dunod.

Gumuchian H., Grasset E., Lajarge R., Roux E., 2003. Les acteurs, ces oubliés du territoire, Paris, Anthropos.
Lalubie G., Gros-Désormeaux J.-R., Nicolas T., 2015. L'application de la DCE dans les départements et régions d'outre-mer: la mise en lumière des discordances entre le concevable et le possible à la Martinique, Norois, 235, 2, 51-66, www.cairn.info/revue-norois-2015-2-page-51.htm.

Le Quino A., 2017. Débat public, in Kada N., Pasquier R., Courtecuisse C., Aubelle V. (Eds), Dictionnaire encyclopédique de la décentralisation, Boulogne-Billancourt, Berger-Levrault, 355-359.

Lequin M., 2009. Création d'une aire protégée et logiques d'action de l'état et du milieu : analyse d'une irréconciabilité constructive à l'œuvre, Études caribéennes, 12, doi: 10.4000/etudescaribeennes.3565.

Micoud A. (Ed.), 1991. Des hauts-lieux. La construction sociale de l'exemplarité, Paris, CNRS Éditions.

Pissaloux J.-L., 2011. La démocratie participative dans le domaine environnemental, Revue française d'administration publique, 137-138, 1-2, 123-137, doi:10.3917/ rfap.137.0123.

Reynal L., Volny-Anne C., Pau C., Greaux S., Simmonet L., Brin-Desnoue W., Precheur C., Garnier J., Blanchard F., Lespagnol P., Depetris M., Weiss J., Demaneche S., Le Blond E., Leblond C., Merrien C., Le Rû C., Daurès F., Berthou P., 2016. Situation de la pêche en Martinique en 2014. Fiche de synthèse, Fort de France, Ifremer.

Richard A., 2015. Démocratie environnementale : débattre et décider. Rapport de la Commission spécialisée du Conseil national de la transition écologique sur la démocratisation $d u$ dialogue environnemental, Paris, ministère de l'Écologie, du Développement durable et de l'Énergie.

Ricœur P., 1969. Le conflit des interprétations. Essais d'herméneutique, Paris, Éditions du Seuil.

Rostaing T., 2014. Quel avenir pour la pêche martiniquaise à l'horizon 2020 : lignes directrices pour l'action de la direction de la Mer et outils financiers à mobiliser, Fort-de-France, École d'administration des affaires maritimes.

Rostaing T., Raveau A., Rynikiewicz C., Degaulejac B., 2016. Économie bleue en Martinique : rapport final, Fort-deFrance, direction de la Mer (Martinique)/Acteon/Creocean/ SCE.

Santos C., 2011. ¿Qué protegen las áreas protegidas? Conservación, producción, Estado y sociedad en la implementación del Sistema Nacional de Áreas Protegidas, Montevideo, Trilce.

Spotorno M., 2005. Le Parc naturel régional de Portofino en Ligurie, Méditerranée, 105, doi: 10.4000/mediterra nee.342.

Thirot M., Failler P., Daniel J., 2017. Des savoirs locaux aux experts naturalistes, Revue d'ethnoécologie, 11, doi: 10.4000/ethnoecologie.2916.

Vaillant L., 2016. Monographie maritime 2015-2016 de la Martinique. Rapport, Fort-de-France, direction de la Mer (Martinique).

Van Tilbeurgh V., Charlier-Kerbiguet M., 2009. La difficile gestion d'un parc marin, in Berlan-Darqué M., Larrère R., Lizet B. (Eds), Histoire des parcs nationaux. Comment 
prendre soin de la nature?, Versailles/Paris, Quæ/Muséum national d'histoire naturelle, 111-128.

Weigel J.-Y., Féral F., Cazalet B. (Eds), 2007. Les aires marines protégées d'Afrique de l'Ouest. Gouvernance et politiques publiques, Perpignan, Presses universitaires de Perpignan.

Zarka J.-C., 2016. La Charte de la participation du public, Petites affiches, 246, 6.

Citation de l'article : Mazurek H., Arreghini L., Cochet C., Daniel J., Gros-Désormeaux J.-R., Pereira D., 2019. La création du parc naturel marin de Martinique: entre concertation et fragmentation. Nat. Sci. Soc. 27, 3, 310-326. 